\title{
Du laurier dans les greniers de Grande Canarie
}

Insecticide naturel et conservation longue des récoltes à l'époque préhispanique

Jacob Morales, Pedro Henríquez-Valido, Marco Moreno-Benítez, Yurena Naranjo-Mayor et Amelia Rodríguez-Rodríguez

Traducteur : Valentine Leÿs

\section{OpenEdition \\ Journals}

Édition électronique

URL : https://journals.openedition.org/tc/8930

DOI : $10.4000 /$ tc. 8930

ISSN : $1952-420 X$

Éditeur

Éditions de l'EHESS

Édition imprimée

Date de publication : 17 mai 2018

Pagination : 126-129

ISBN : 978-2-7132-2750-9

ISSN : 0248-6016

\section{Référence électronique}

Jacob Morales, Pedro Henríquez-Valido, Marco Moreno-Benítez, Yurena Naranjo-Mayor et Amelia Rodríguez-Rodríguez, "Du laurier dans les greniers de Grande Canarie », Techniques \& Culture [En ligne], 69 | 2018, mis en ligne le 17 mai 2020, consulté le 29 septembre 2022. URL : http:// journals.openedition.org/tc/8930; DOI : https://doi.org/10.4000/tc.8930 


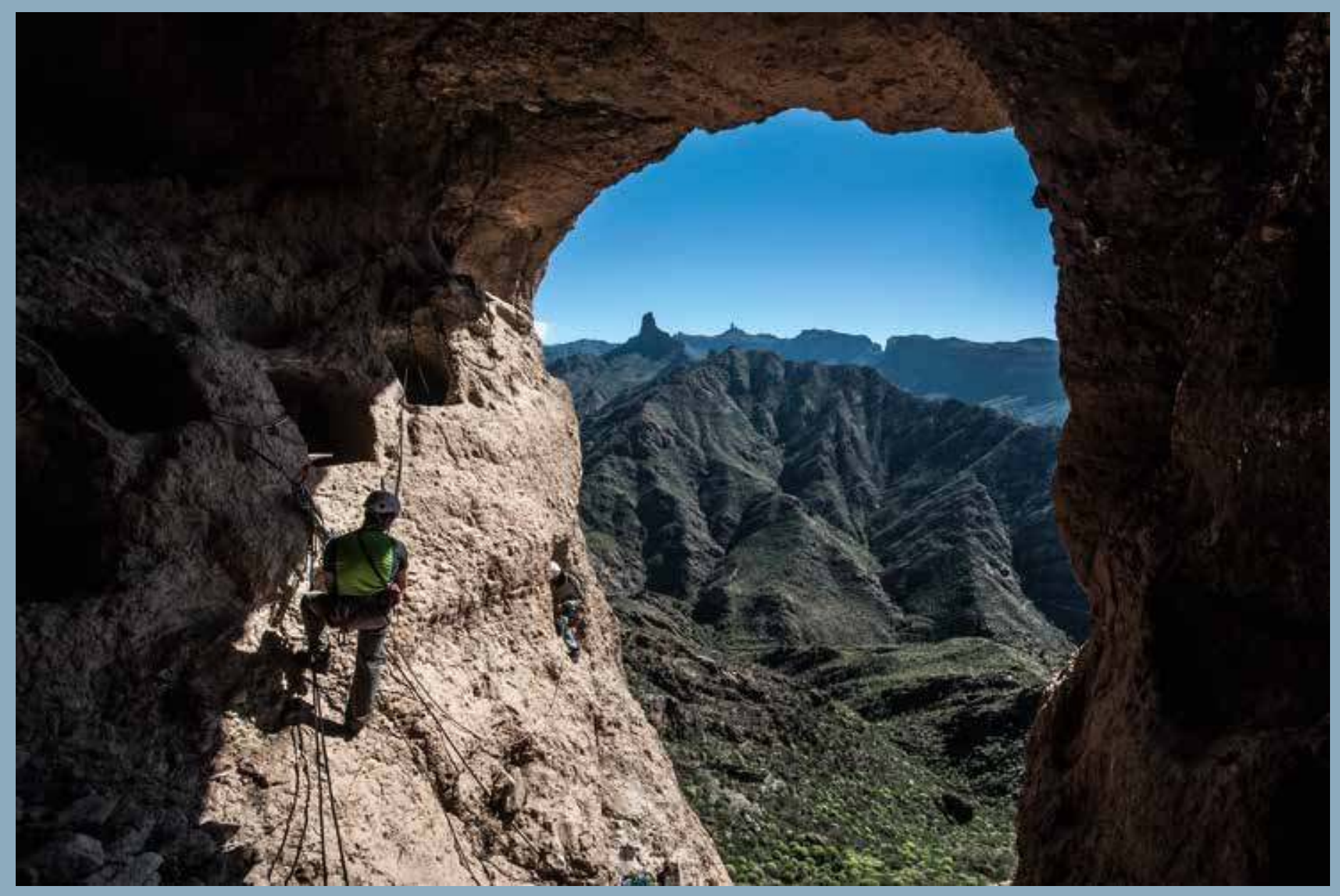




\section{Du laurier dans les greniers de Grande Canarie}

\section{Insecticide naturel et conservation longue des récoltes à l'époque préhispanique}

Les insectes constituent l'un des principaux problèmes rencontrés lorsqu'il s'agit de stocker sur le long terme des aliments, en particulier des céréales. On estime qu'avant l'emploi systématique d'insecticides chimiques, les insectes endommageaient au moins 10 à 20\% du grain stocké -lorsqu'ils n’altéraient pas la totalité de la récolte. Des documents historiques témoignent de l'utilisation de produits organiques et inorganiques pour éliminer ou chasser les insectes. En revanche, les données archéologiques sur cet aspect essentiel du stockage sont rares, ce qui s'explique en partie par le manque de découvertes d'aliments anciens conservésin situ. Quelles méthodes et techniques ont été utilisées dans le passé pour stocker les denrées et éviter les infestations par les insectes? Pour répondre à cette question, nous présentons les résultats préliminaires d'une étude conduite dans le cadre d'un projet pluridisciplinaire sur un ensemble de greniers de l'île de la Grande Canarie (îles Canaries, Espagne) datant de la période pré-hispanique (environ 500-1500 apr. J.-C.). Les populations autochtones de Grande Canarie étaient des agriculteurs, qui tiraient principalement leur subsistance des plantes cultivées et qui ont construit de grands greniers collectifs pour leur stockage. Notre étude examine six greniers collectifs (Cenobio de Valerón, La Montañeta, El Álamo-Acusa, Cuevas Muchas-Guayadeque, Risco-Pintado-Temisas et La Fortaleza) ainsi que leur contenu, en s'appuyant sur les méthodes de l'archéobotanique et de l'archéoentomologie ainsi que celles de l'analyse morpho-technique et fonctionnelle des outils de pierre et de l'analyse spatiale. Les greniers se composent de groupes de silos situés dans des lieux difficiles d'accès, comme des falaises. Les greniers et leurs silos étaient creusés dans un tuf volcanique, une roche qui maintient un niveau constant de température et d'humidité. On peut attester aussi que les fissures des parois étaient de plus revêtues d'une épaisse couche de mortier pour améliorer leur niveau d'isolation. Il est intéressant de noter que les greniers collectifs de Grande Canarie sont semblables aux igoudar, ou «magasins de falaise» des populations berbères du Maroc et d'autres régions du Maghreb (Onrubia-Pintado 1995).

Les résultats des analyses spatiales indiquent que la localisation des greniers était déterminée avant tout par la présence de falaises d'accès difficile. Ce choix répondait à la nécessité de protéger 
7. Restes archéologiques des principaux types de végétaux et d'insectes trouvés dans les greniers a) orge, enveloppes articulées (El ÁlamoAcusa); b) blé, grains (La Fortaleza); c) fève, hile et fragments de tegulum (La Fortaleza); d) lentille, graine; e) figue, graines (Cenobio de Valerón); f) feuille de laurier des Canaries, fragment (Cuevas Muchas-Guayadeque); g) charançons du blé (El Álamo-Acusa) ces installations contre les animaux et les autres groupes d'humains. En second lieu, la situation des greniers est conditionnée par leur proximité par rapport aux meilleures terres agricoles. Nos données suggèrent que les greniers se trouvaient éloignés des champs de 30 à 45 minutes à pied. En ce qui concerne l'étude des outils de pierre, nos résultats indiquent que les greniers étaient aussi utilisés pour la transformation des plantes stockées dans les silos. Parmi les outils de meulage relevés dans les greniers, on compte des moulins à bras, des meules dormantes et des mortiers, ainsi que des molettes et des pilons. Un nombre significatif d'outils de meulage porte des traces d'usure liées au traitement des végétaux, ce qui suggère que cette activité était fréquente dans les greniers. Ces assemblages comprennent aussi d'autres outils de pierre qui indiquent que diverses tâches, notamment l'entretien des silos y était effectué. De nombreux restes de plantes (103975) ont été relevés dans les différents greniers. Nous avons identifié en tout six espèces de plantes cultivées: l'orge à six rangs (Hordeum vulgare, sous-espèce vulgare), le blé dur (Triticum durum), la lentille (Lens culinaris), la fève (Vicia faba), le pois mange-tout (Pisum sativum) et la figue (Ficus carica). Nous avons par ailleurs relevé des plantes sauvages qui étaient elles aussi stockées dans les silos, parmi lesquelles le laurier des Canaries (Laurus cf. novocanariensis), le pin des Canaries (Pinus canariensis), le «mocán» (Visnea mocanera), l'arbre au mastic (Pistacia lentiscus) et le dattier des Canaries (Phoenix canariensis). Cependant, l'orge et les figues sont les restes les plus communément relevés. Ces analyses de restes végétaux prélevés dans un contexte domestique aussi bien que les analyses de diète d'ossements humains concordent avec les documents ethnohistoriques qui indiquent que l'orge et la figue formaient la base du régime des populations pré-hispaniques de Grande Canarie. Les preuves trouvées dans les silos, et notamment un grand nombre d'épis fragmentés et complets portant des marques de coupe à la base, suggèrent que l'orge et le blé étaient stockés sous forme d'épis. Par ailleurs, nos données indiquent que les légumineuses étaient stockées dans leur cosse pour prévenir les infestations d'insectes. Cette méthode de stockage était plus avantageuse pour les céréales et les légumineuses, car les graines étaient ainsi naturellement protégées par leur enveloppe. On rencontre cependant des preuves de la présence d'insectes nuisibles dans la plupart des échantillons, malgré l'usage de différentes méthodes de stockage par les populations pré-hispaniques. Des grains endommagés et des insectes ont été trouvés dans tous les greniers sauf celui de La Montañeta. Bien que des analyses soient en cours, de nombreux restes (au moins 6624) de 
charançons du blé (Sitophilus granarius) ont été identifiés. S. granarius est considéré comme nuisible pour le stockage des denrées: en effet, cet insecte est un sinanthrope qui vit et ne se reproduit que dans les stocks de grains. Le nombre élevé de charançons dans les silos indique que ces insectes représentaient un problème considérable pour le stockage à long terme. Pour repousser ces insectes de leurs silos, les populations indigènes de Grande Canarie utilisaient des plantes insecticides. Des fragments desséchés de laurier des Canaries ont été identifiés dans les greniers d'El Álamo, Cuevas Muchas et Temisas. Différentes datations au carbone 14 des fragments de feuilles indiquent que ces dernières faisaient partie du contenu originel des silos. Lanalyse biochimique d'un composé de ces feuilles a confirmé que leurs huiles réduisent la consommation des denrées par les insectes, ont un fort effet fongicide et inhibent la germination des graines (Rodilla et al. 2008). Nous interprétons donc la présence de feuilles de laurier des Canaries dans les greniers de l'île comme un moyen de repousser les insectes. Il s’agit à notre connaissance de la première preuve archéologique directe de l'usage de plantes insecticides dans le cadre du stockage des aliments.

\section{En ligne}

Retrouver l'article complet sur journals.openedition.org/tc: TechniquesECulture 69 «Le temps des aliments».

\section{Iconographie}

Image d'ouverture. Vue de l'accès au second niveau du grenier d'El Álamo-Acusa. @ E. Martín. 1. C J. Morales.

\section{Les auteurs}

Jacob Morales est chercheur au G.I. Tarha de l'université de Las Palmas de Gran Canaria (Espagne). Ses recherches portent sur la transition vers l'agriculture en Afrique du Nord, l'adaptation de l'agriculture en milieu insulaire, et plus largement les interactions homme-plantes. Pedro Henríquez-Valido, doctorant au G.I. Tarha (ULPGC), étudie le stockage pendant la période préhispanique des îles Canaries en combinant des approches en archéoentomologie et en archéobotanique. Marco Moreno-Benítez est consultant archéologique et directeur de Tibicena, Arqueología y Patrimonio. Ses recherches portent sur les analyses spatiales et l'utilisation des SIG. Yurena Naranjo-Mayor est doctorante au G.I. Tarha (ULPGC). Ses recherches portent sur l'utilisation d'outils en pierre dans la préhistoire, en particulier sur les outils de broyage des îles Canaries. Amelia Rodríguez-Rodríguez est professeur de Préhistoire au G.I. Tarha (ULPGC). Ses recherches portent sur l'utilisation d'outils en pierre et la technologie lithique avec un accent particulier sur les analyses morpho-techniques et fonctionnelles dans différents contextes géographiques et historiques.

\section{Références}

Onrubia-Pintado, J. 1995 «Magasins de falaise pré-hispaniques de la Grande Canarie. Viabilité et conditions de formulation d'une hypothèse de référence ethnoarchéologique» in A. Bazzana \& M.-C. Delaigue dir. Ethnoarchéologie méditerranéenne, finalités, démarches et résultats. Madrid: Casa de Velásquez: 159-180.
Rodilla, J. M., Tinoco, M. T., Morais, J. C., Gimenez, C., Cabrera, R. et al. 2008 «Laurus novocanariensis essential oil: Seasonal variation and valorization», Biochemical Systematics and Ecology 36: 167-176.

\section{Pour citer l'article}

Morales, J., Henríquez-Valido, P., Moreno-Benítez, M., Naranjo-Mayor, Y. \& A. Rodríguez-Rodríguez 2018 « Du laurier dans les greniers de Grande Canarie (Espagne). Insecticide naturel et conservation longue des récoltes à l'époque préhispanique», TechniquesE Culture 69 «Le temps des aliments», p. 126-129. 